\title{
Experimental Study of Vibrational and Pure Rotational Coherent Anti-Stokes Raman Scattering (CARS) in Molecular Hydrogen
}

\author{
G. Marowsky, A. Gierulski, and B. Dick \\ Max-Planck-Institut für biophysikalische Chemie, Abteilung Laserphysik, \\ D-3400 Göttingen, Fed. Rep. Germany \\ U. Sowada and R. Vehrenkamp \\ Lambda Physik GmbH, P.O. Box 2663, D-3400 Göttingen, Fed. Rep. Germany \\ Received 23 May 1985/Accepted 24 August 1985
}

\begin{abstract}
Using a specially designed excimer-laser-pumped dye laser of adjustable bandwidth high-lying pure rotational transitions of both, ortho- and para-hydrogen have been identified by coherent anti-Stokes Raman scattering (CARS). As an interesting application $\mathrm{H}_{2}$-based CARS-thermometry is discussed.
\end{abstract}

PACS: 42.65

Coherent anti-Stokes Raman spectroscopy (CARS) using rotational-vibrational transitions has proven to be a powerful tool in various areas of science and technology since its first demonstration by Maker and Terhune [1]. Use of pure rotational transitions, however, has been made so far only in a few applications dealing with concentration and temperature measurements of small molecules, such as $\mathrm{H}_{2}, \mathrm{~N}_{2}, \mathrm{O}_{2}, \mathrm{~N}_{2} \mathrm{O}$, and $\mathrm{CO}$ [2-12]. It is the purpose of this paper to demonstrate that pure rotational CARS spectroscopy in the vibronic groundstate of molecular hydrogen is a very powerful tool for the detection of small concentrations of $\mathrm{H}_{2}$, which can be utilized effectively for temperature measurements in various temperature ranges.

The principal difference in terms of energy levels used for rotational and vibrational CARS of hydrogen can be explained with reference to Fig. 1, showing the typical scattering processes taking place in a CARStype experiment. The striking difference between vibrational and rotational CARS is the energy difference between the respective resonantly excited real levels, such as $\Delta E_{\mathrm{vib}}$ for the $v=0 \rightarrow v=1$ transition and $\Delta E_{\text {rot }}$ for the $J=1 \rightarrow J=3$ transition, which turned out to be the most intense rotational transition for $\mathrm{H}_{2}$ trace analysis under room temperature conditions. The limits of detectibility will be discussed in detail in Sect. 2.

\section{Broadband Dye Laser}

CARS spectra for concentration and temperature analysis are usually recorded by scanning the Stokes frequency $\omega_{2}$ across a real vibrational or rotational state of the system under consideration (Fig. 2a) and observing the anti-Stokes emission of frequency $\omega_{3}=\omega_{A}$ with a monochromator. An experimental alternative has been described by Roh et al. [13]. A broad-band dye laser of bandwidth $\Delta \omega$ is used in conjunction with a narrow-band pump laser to generate an entire anti-Stokes spectrum of transitions using a single laser pulse (Fig. 2b). Application of this technique resulted in the instantaneous generation of an entire $Q$-branch spectrum of molecular hydrogen. 


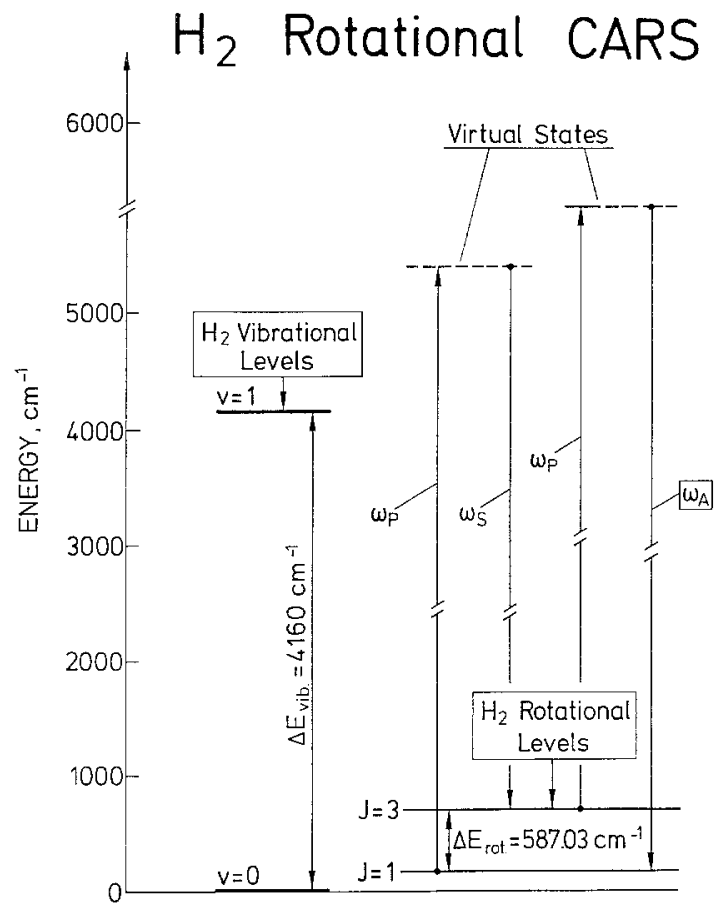

Fig. 1. Energy levels for rotational and, for comparison, vibrational transitions used for CARS experiments. $\omega_{P}$ and $\omega_{S}$ denote frequency of pump and Stokes laser, $\omega_{A}$ frequency of anti-Stokes emission. As example rotational levels of $J=1 \rightarrow J=3$ transitions are indicated

(a) Scanning-CARS

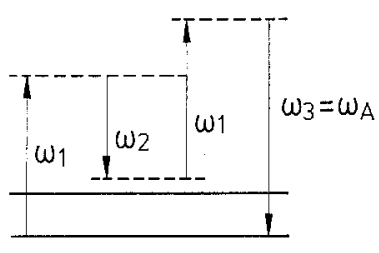

(b) Multiplex-CARS

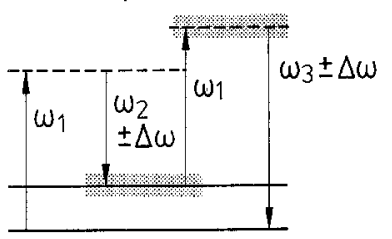

Fig. 2. (a) Energy levels for narrow-band scanning CARS technique, (b) schematic of multiplex CARS using a broad-band laser of halfwidth $\Delta \omega$ as Stokes laser

For optimized signal detection the bandwidth $\Delta \omega$ should just cover the frequency range of interest. The excimer-laser-pumped dye laser shown in Fig. 3 allows a convenient choice of the required bandwidth by adjusting the wavelength-selective grating to the ap-
BROADBAND DYE LASER FL 1002 C

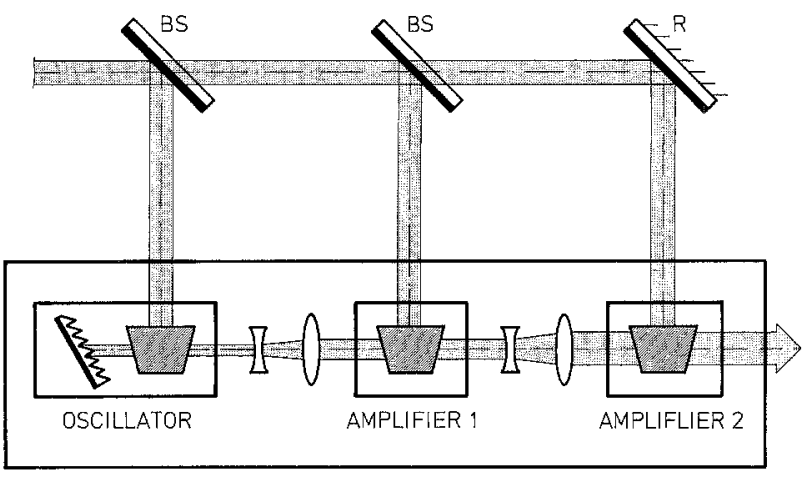

Fig. 3. Optical schematic of Lambda Physik broad-band dye laser type FL $1002 \mathrm{C}$
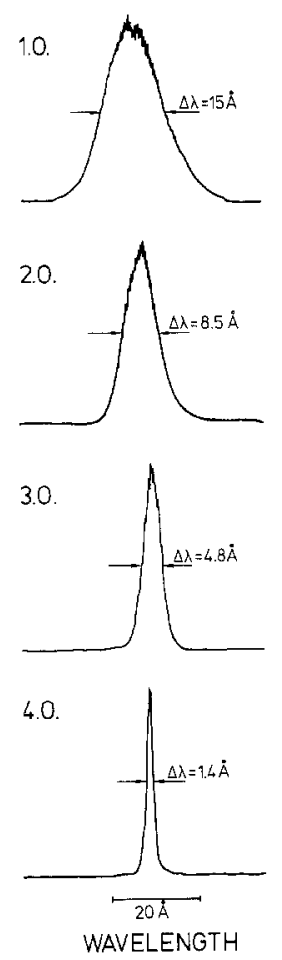

Fig. 4. Output spectra of broad-band dye laser obtained with four different settings of the oscillator diffraction grating $(1.0 \bumpeq$ first order etc.)

propriate order in retro-reflection. The oscillator of this laser consists only of a transversely pumped dye cell and a diffraction grating. Avoiding any normal glass surfaces, the radiation leaving the oscillator subsequently passes through a two-stage amplifier system. With an input energy of $100 \mathrm{~mJ}$ at the wavelength of $\mathrm{XeCl}(308 \mathrm{~nm})$ the conversion efficiency in the red spectral range is typically $10 \%$. Figure 4 shows a series of laser output spectra obtained by rotation of 
the oscillator grating from first to fourth order, resulting in a variation of bandwidths from 15 to $1.4 \AA$. This laser has been used to examine one particular $Q$ branch transition of hydrogen at room temperature. Figure 5 shows for comparison an entire $Q$-branch spectrum (upper trace) of $\mathrm{H}_{2}$ at a pressure of 100 Torr together with a $Q_{1}$-spectrum at 2.5 Torr, taken with a Stokes-laser bandwidth reduced by a factor of 10 . Taking into account the decrease in $\mathrm{H}_{2}$-density by a factor 40, the signal-intensity of $Q_{1}$ in the lower trace reflects an increase in spectral brightness by a factor by 10 . The spectrum of $Q_{1}$, shown for clarity on an expanded scale (500 vidicon channels $\cong 75 \mathrm{~cm}^{-1}$ ), clearly indicates that with the narrowest available bandwidth $\left(4.2 \mathrm{~cm}^{-1}\right)$ of the Stokes-laser no neighbouring $Q$-branch transitions are excited. In fact, according to $[14]$ the spacing between $Q_{1}$ and $Q_{2}$ is $12 \mathrm{~cm}^{-1}$. Rejection of the other transitions is an experimental indication that the Stokes-laser emission is sharply restricted to the bandwidth indicated. This avoids increase of the background level due to the short-wavelength tail of the dye fluorescence, which is a problem encountered in rotational CARS spectroscopy, when using a free-running dye laser with no intracavity wavelength-selective elements.

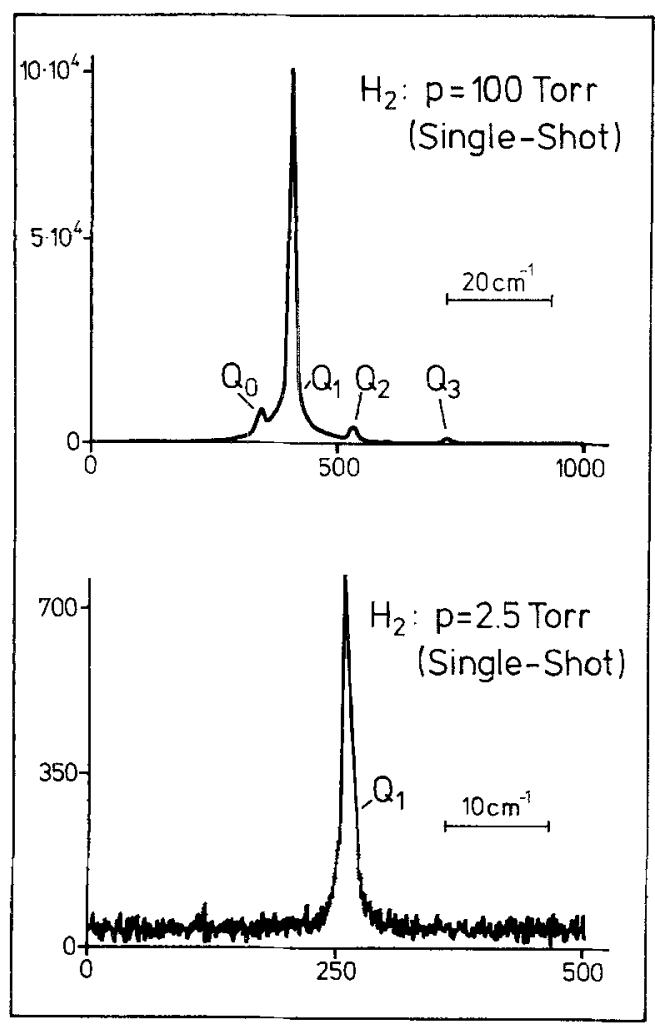

Fig. 5. Selection of $Q_{1}$ from $\mathrm{H}_{2} Q$-branch spectrum by appropriate reduction of broad-band dye-laser bandwidth

\section{Experimental Set up}

The experimental arrangement (Fig.6) allowed the demonstration of visible CARS signals with the $\mathrm{H}_{2}$-cell operated at pressures in the atm-range and detection of low-density signals in the mTorr-range. Visual inspection was facilitated by a narrowband interference filter $(\Delta \lambda=50 \AA)$ rejecting part of the intense pumpand Stokes-laser radiation and a dispersive grating for spatial separation of the collinear beams of wavelengths $\lambda_{p}, \lambda_{S}$, and $\hat{\lambda}_{\mathrm{AS}}$. The registration system for low $\mathrm{H}_{2}$-pressures consisted of a monochromator (Jobin Yvon $1 \mathrm{~m}$ double-monochromator) together with a triggered diode-array camera (OMA-III of PAR). Best results were obtained with a narrow-band dye laser (type FL 2002) and a broad-band dye laser (type FL 1002C). Both lasers are pumped with an excimer laser (type EMG 201 of $300 \mathrm{~mJ}$ typical pulse energy at $308 \mathrm{~nm}$ with $\mathrm{XeCl}$ ). The dye lasers delivered 20 and $10 \mathrm{~mJ}$, respectively, for pump and Stokes laser. The radiation of these lasers was combined by means of a dichroic beam splitter and focused with a lens of $30 \mathrm{~cm}$ focal length into the $\mathrm{H}_{2}$-cell. Figure 7 summarizes the relevant spectral data of pump, Stokes, and anti-Stokes wavelengths used in the course of these collinear, rotational CARS studies. For efficient rejection of the excitation radiation all experiments were centered around the transmission of a narrow-band interference filter that transmits in combination with the double-monochromator a spectral separation of the excitation wavelengths as low as

$\left|\lambda_{P}-\hat{\lambda}_{S}\right|_{\min } \geqq 50 \AA$

A separation of $50 \AA$ or $150 \mathrm{~cm}^{-1}$ in this spectral range is sufficient for all rotational CARS studies with hydrogen. Table 1 lists the lowest rotational transitions in the vibronic groundstate of hydrogen [14]. The spectral separation of $50 \AA$ is presumably too large for larger molecules such as $\mathrm{CO}, \mathrm{O}_{2}$, and $\mathrm{N}_{2}$. Their rotational CARS analysis requires more-dimensional arrangements of the beam geometry as BOXCARS or even folded BOXCARS [15-17]. Due to the rather high signal levels no provisions were made to suppress the non-resonant background contribution by

Table 1. Transition energies of the lowest 6 rotational transitions $J \rightarrow J+2$ in hydrogen [14]

\begin{tabular}{lr}
\hline$J=0 \rightarrow J=2$ & $354 \mathrm{~cm}^{-1}$ \\
$J=1 \rightarrow J=3$ & $587 \mathrm{~cm}^{-1}$ \\
$J=2 \rightarrow J=4$ & $814 \mathrm{~cm}^{-1}$ \\
$J=3 \rightarrow J=5$ & $1035 \mathrm{~cm}^{-1}$ \\
$J=4 \rightarrow J=6$ & $1246 \mathrm{~cm}^{-1}$ \\
$J=5 \rightarrow J=7$ & $1448 \mathrm{~cm}^{-1}$ \\
\hline
\end{tabular}




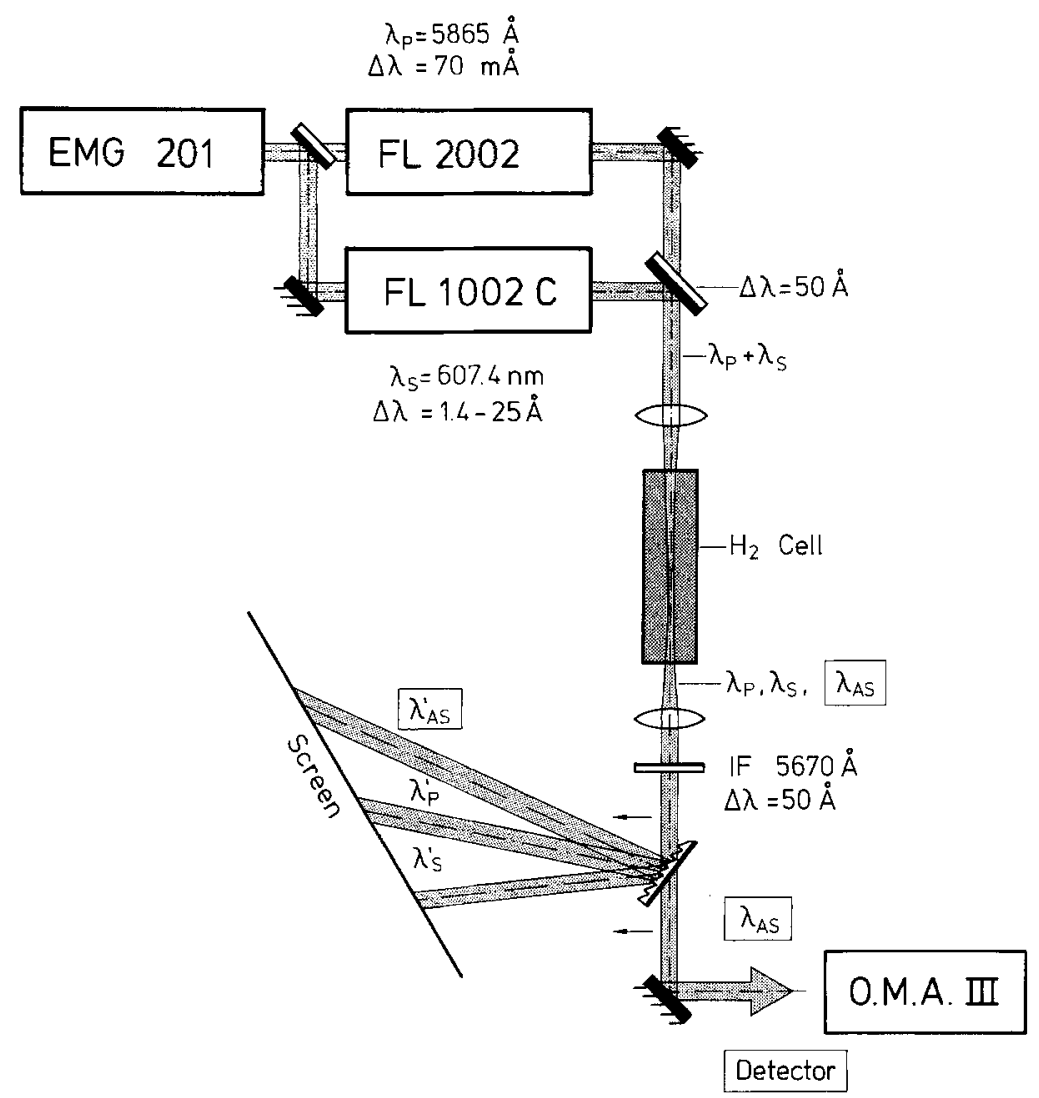

Fig. 6. Experimental setup for rotational $\mathrm{H}_{2}$ CARS studies, allowing both, visual inspection of anti-Stokes radiation at a screen and sensitive detection with OMA-III diode-array camera after removal of diffraction grating

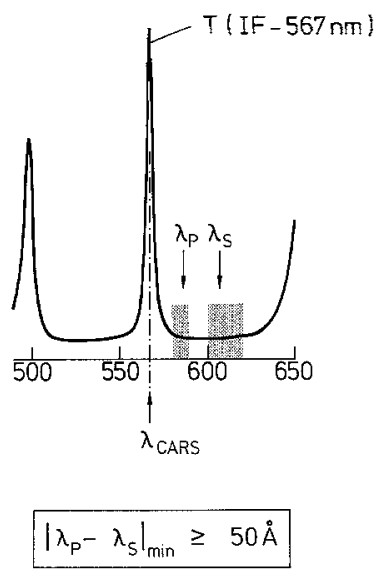

Fig. 7. Transmission characteristics of narrow-band interference filter centered at $5670 \AA$ and spectral operation range of pump and Stokes laser

polarization-sensitive detection and appropriate polarization of the excitation beams [18-20]. The performance of the detection system in terms of linewidth is depicted in Fig. 8. The linewidth of the narrow-band pump laser (FL 2002) of $0.07 \AA$ is beyond the resolving power of the monochromator-vidicon combination, yielding a technical width of $0.1 \AA$. When excited with a Stokes laser of $1.4 \AA$ bandwidth, the width of the rotational CARS transitions is typically $0.2 \AA$ and is also shown in Fig. 8 .

\section{Experimental Results}

Relatively rare transitions of para-hydrogen and low $\mathrm{H}_{2}$-concentrations were observed. In the literature so far only the $J=3 \rightarrow J=5$ and the $J=1 \rightarrow J=3$ transition $[4,5]$ have been characterized by anti-Stokes Raman scattering. ${ }^{1}$ So far nothing has been reported concerning the para-hydrogen species [21] with antiparallel nuclear spin orientation. In addition, CARS signals are expected to be rather low due to the low concentration of para-hydrogen at room temperature. As an experimental result, all transitions indicated in Table 1 have been observed with CARS spectroscopy. With the excitation conditions, as described in Sect.2, and a total $\mathrm{H}_{2}$-pressure of 100 Torr the rotational transitions $J=4 \rightarrow J=6$ and $J=5 \rightarrow J=7$ could still be monitored (Fig.9). Even higher transitions, such as $J=9 \rightarrow J=11$ which coincides with the fundamental, vibrational CARS mode of carbonmonoxide at $2131 \mathrm{~cm}^{-1}$ are unavailable under room temperature conditions but have been recently observed in connection with combustion studies [5]. Table 2 summarizes limits of detection obtained for $\mathrm{H}_{2}$ using the rotational

\footnotetext{
1 Stimulated rotational Raman scattering in para-hydrogen is an efficient means of generating coherent radiation in the $13-18 \mu \mathrm{m}$ region [25]
} 


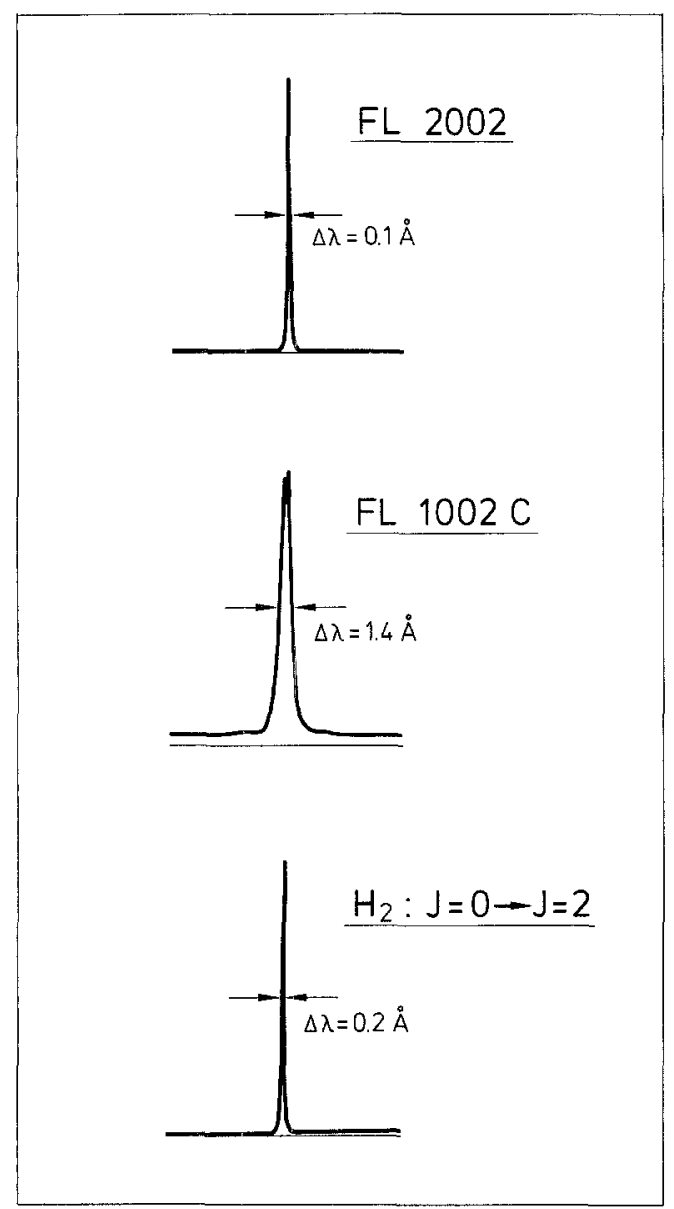

Fig. 8. Output spectra of the FL 2002 and FL $1002 \mathrm{C}$ lasers together with $J=0 \rightarrow J=2$ rotational CARS line

$J=1 \rightarrow J=3$ transition and the $Q_{1}$-band of the vibrational branch for comparison.

The first row refers to single-shot spectra with subtraction of the background due to electrical noise of the diode-array camera and due to scattering light in the detection system. Long time averaging refers to collection of up to $10^{4}$ laser shots.

The apparent difference in sensitivity between rotational and vibrational transitions (Table 2) can be explained in terms of the resonant enhancement of the nonlinear susceptibility $\chi^{(3)}$. The generated power of the CARS signal is proportional to the square of the pump laser intensity $I_{1}$, the Stokes-laser intensity $I_{2}$, and the square modulus of the susceptibility $\chi^{(3)}$

$P^{\mathrm{CARS}} \sim I_{1}^{2} \cdot I_{2} \cdot\left|\chi^{(3)}\right|^{2}$.

Close to resonance the susceptibility is given by

$$
\begin{aligned}
\chi^{(3)}= & \frac{N}{h} \sum_{a, b}\left(\varrho_{a}-\varrho_{b}\right) \frac{c^{4}}{\omega_{1} \omega_{2}^{3}}\left(\frac{d \sigma}{d \Omega}\right)_{a b} \\
& \frac{1}{\omega_{b a}-\omega_{1}+\omega_{2}+\mathrm{i} \Gamma_{a b}} .
\end{aligned}
$$
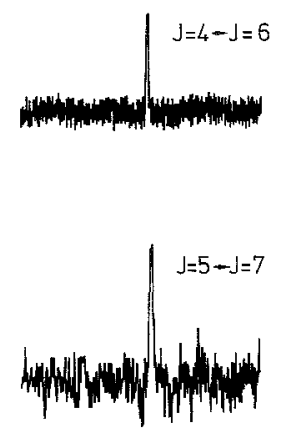

Fig. 9. Rotational CARS spectra of vibrational ground state

In this expression $N$ is the number density, $d \sigma / d \Omega$ the Raman cross section for the transition $|a\rangle \rightarrow|b\rangle, \varrho_{a}$ the population of level $|a\rangle$, and $\Gamma_{a b}$ the Raman linewidth.

For low concentrations at room temperature the linewidth is dominated by the Doppler width $\Gamma_{D}$ which is proportional to the Stokes shift. Thus on resonance,

$\left|\chi^{(3)}\right| \sim 1 / \Gamma_{D} \sim \frac{1}{\omega_{1}-\omega_{2}}$.

Therefore, rotational CARS resonances $\left(\omega_{1}-\omega_{2}\right.$ $=587 \mathrm{~cm}^{-1}$ ) should be enhanced by almost two orders of magnitude with respect to vibrational CARS resonances $\left(\omega_{1}-\omega_{2}=4160 \mathrm{~cm}^{-1}\right)$ due to the linewidth. In addition, pure rotational Raman cross sections are usually much larger than those for vibrational $Q$ branch transitions. On the other hand, the population difference factor might be smaller for rotational transitions. For a purely rotational transition $J \rightarrow J+2,(2)$ must be summed over all degenerate levels of the initial and final state. For thermal equilibrium the $J$ dependent part of the susceptibility is

$$
\begin{gathered}
\chi^{(3)}(J \rightarrow J+2) \sim \frac{N}{Q(T)} \cdot \frac{(J+1)(J+2)}{(2 J+3)} \\
\cdot[X(J, T)-X(J+2, T)], \\
X(J, T)=g_{J} \cdot \exp [-E(J) / k T), \\
Q(T)=\sum_{J, v} g_{J}(2 J+1) \exp [-E(J, v) / k T], \\
E(J, v)=E_{v}^{0}+B_{v} J(J+1)+D_{v} J^{2}(J+1)^{2} \\
+H_{v} J^{3}(J+1)^{3} .
\end{gathered}
$$

Table 2. Detection limits for $\mathrm{H}_{2}$ with rotational and vibrational CARS with the apparatus used

\begin{tabular}{lcr}
\hline & Rot. CARS & Vib. CARS \\
\hline $\begin{array}{l}\text { Single shot } \\
\begin{array}{c}\text { Long-time } \\
\text { averaging }\end{array}\end{array}$ & $\begin{array}{r}10 \mathrm{~m} \text { Torr } \\
\mathrm{mT} \text { Torr }\end{array}$ & $\begin{array}{r}100 \mathrm{~m} \text { Torr } \\
10 \mathrm{mTorr}\end{array}$ \\
\hline
\end{tabular}


Here $B_{v}, D_{v}$, and $H_{v}$ are the rotational constants in the vibrational state $v$ with vibrational energy $E_{v}^{0}$, and $g_{J}$ is the nuclear spin weight:

$g_{J}= \begin{cases}3 & \left.\text { for } J \text { odd (ortho- } \mathrm{H}_{2}\right) \\ 1 & \left.\text { for } J \text { even (para- } \mathrm{H}_{2}\right)\end{cases}$

The conversion between ortho- and para-hydrogen is spin-forbidden and slow in the absence of a catalyst. Therefore, after a change of temperature, the system behaves as a mixture of two species with their own total populations and partition functions $Q(T)$. In this case $N$ in (4) must be replaced by $N_{0}$ or $N_{p}$, and the sum in $Q(T)$ runs over odd $J$ or even $J$ for ortho- or parahydrogen, respectively.

In the intensity ratio of two transitions both in orthoor both in para- $\mathrm{H}_{2}$ the partition functions and population factors cancel and the temperature can be determined.

The intensity ratio of a transition in ortho- $\mathrm{H}_{2}$ and in para- $\mathrm{H}_{2}$ contains the population ratio $N_{0} / N_{p}$ as an additional variable which can be determined when the temperature is known. This could be used to check whether equilibrium between both modifications has been achieved at the new temperature, or to follow the kinetics of the ortho $\leftrightarrow$ para conversion. A thermometer based upon pure rotational transitions is represented in Fig. 10. The intensities of the lowest 6 transitions are shown as a function of temperature for a $\mathrm{H}_{2}$-system in thermal equilibrium. The whole temperature range depicted in Fig. 10 is experimentally accessible from the standpoint of detection sensitivity. Each pair of transitions constitutes a potential CARS

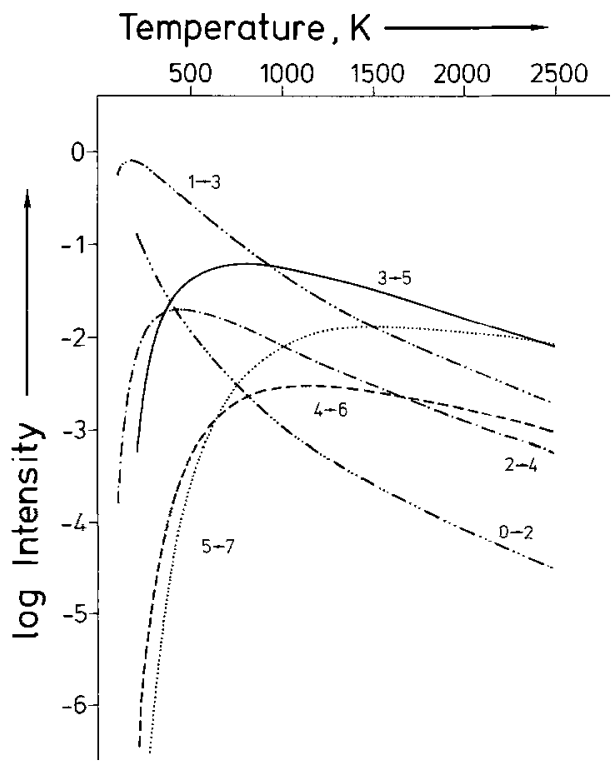

Fig. 10. Intensities of the lowest 6 rotational CARS transitions as a function of temperature. A Boltzmann distribution of populations was assumed. Rotational constants were taken from [14] thermometer. The best choice are transitions with strongly different slopes in Fig. 10. The pair $0 \rightarrow 2 / 2 \rightarrow 4$ could be used from room temperature up to ca. $1000 \mathrm{~K}$, the pair $1 \rightarrow 3 / 3 \rightarrow 5$ in the range from 500 to $1500 \mathrm{~K}$, and the pairs $3 \rightarrow 5 / 5 \rightarrow 7$ and $2 \rightarrow 4 / 4 \rightarrow 6$ in the whole range up to $2500 \mathrm{~K}$. All these thermometers do not depend on the relative population of the ortho- and para- $\mathrm{H}_{2}$ level systems. However, they have the disadvantage that the transitions involved are spectrally far apart. That makes it difficult to measure the intensities under identical conditions, since interference filters, adjustment and even the laser dye would have to be changed.

An experimental alternative would be to monitor simultaneously a $\triangle J=2$ transition in the $v=0$ and $v=1$ band. The spectral separation is as follows [14]:

$J=0 \rightarrow J=2: 17.7 \mathrm{~cm}^{-1}$,

$J=1 \rightarrow J=3: 29.4 \mathrm{~cm}^{-1}$,

$J=2 \rightarrow J=4: 40.9 \mathrm{~cm}^{-1}$.

Thus each pair is easily accessible by the multiplex method, provided the population is high enough in the first vibronically excited state.

\section{Conclusions}

The first 6 low-lying rotational transitions of orthoand para-hydrogen have been investigated by multiplex coherent anti-Stokes Raman spectroscopy (CARS) using two excimer laser pumped dye lasers. The transition $J=1 \rightarrow J=3$ allows $\mathrm{H}_{2}$ concentration measurements down to $1 \mathrm{mTorr}$, which represents an one order of magnitude improvement compared to vibrational CARS using the $Q_{1}$-mode of the $Q$-branch. A CARS thermometer based upon intensity ratios of individual rotational transitions is proposed.

Acknowledgements. We thank Lambda Physik and Princeton Applied Research for the loan of equipment.

\section{References}

1. P.D. Maker, R.W. Terhune: Phys. Rev. 137, A801 (1965)

2. J.J. Barrett: Appl. Phys. Lett. 29, 722 (1976)

3. I.R. Beattie, T.R. Gilson, D.A. Greenhalgh: Nature 276, 378 (1978)

4. L.P. Goss, J.W. Fleming, A.B. Harvey: Opt. Lett. 5, 345 (1980)

5. D. Klick, K.A. Marko, L. Rimai: Appl. Opt. 20, 1178 (1981)

6. L.E. Harris: Chem. Phys. Lett. 93, 335 (1982)

7. L.E. Harris: Combustion Flame 53, 103 (1983)

8. D.V. Murphy, R.K. Chang: Opt. Lett. 6, 233 (1981)

9. K. Aron, L.E. Harris, J. Fendell: Appl. Opt. 22, 3604 (1983) 
10. K. Aron, L.E. Harris, J. Fendell: Techn. Rep. ARLCD-TR83033; U.S. Army Armament Research and Development Center, Dover, NJ, USA (August 1983)

11. J. Fendell, L.E. Harris, K. Aron: Techn. Rep. ARLCD-TR83048; U.S. Army Armament Research and Development Center, Dover, NJ, USA (December 1983)

12. Jia-biao Zheng, J.B. Snow, D.V. Murphy, A. Leipertz, R.K. Chang: Opt. Lett. 9, 341 (1984)

13. W.B. Roh, P.W. Schreiber, J.P.E. Taran: Appl. Phys. Lett. 29, $174(1976)$

14. U. Fink, T.A. Wiggins, D.H. Rank: J. Mol. Spectrosc. 18, 384 (1965)

15. A.C. Eckbreth: Appl. Phys. Lett. 32, 421 (1978)

16. J.A. Shirley, R.J. Hall, A.C. Eckbreth:Opt. Lett. 5, 380 (1980)

17. J.B. Snow, Jia-biao Zheng, R.K. Chang: Opt. Lett. 8, 599 (1983)
18. J J. Song, G.L. Eesley, M.D. Levenson: Appl, Phys. Lett. 29, 567 (1976)

19. L.A. Rahn, L.J. Zych, P.L. Mattern: Opt. Commun. 30, 249 (1979)

20. T. Dreier, U. Wellhausen, J. Wolftum, G. Marowsky: Appl. Phys. B 29, 31 (1982)

21. G. Herzberg: Molecular Spectra and Molecular Structure, I. Spectra of Diatomic Molecules (Van Nostrand Reinhold, New York 1950)

22. W.M. Tolles, J.W. Nibler, J.R. McDonald, A.B. Harvey: Appl. Spectrosc. 31, 253 (1977)

23. J.W. Nibler, J.R. McDonald, A.B. Harvey: Opt. Commun. 18, 371 (1976)

24. W. Kreutner, W. Stricker, Th. Just: Ber. Bunsenges. Phys. Chem. 87, 1045 (1983)

25. H. Tashiro et al.: Opt. Lett. 10, 80 (1985) 